\title{
Phases of dipolar bosons in a bilayer geometry
}

\author{
Fabio Cinti,,$^{1,2, *}$ Daw-Wei Wang, ${ }^{3,4, \dagger}$ and Massimo Boninsegni ${ }^{5, \ddagger}$ \\ ${ }^{1}$ National Institute for Theoretical Physics (NITheP), Stellenbosch 7600, South Africa \\ ${ }^{2}$ Institute of Theoretical Physics, Stellenbosch University, Stellenbosch 7600, South Africa \\ ${ }^{3}$ Physics Department, National Tsing-Hua University, Hsinchu, Taiwan \\ ${ }^{4}$ National Center for Theoretical Sciences, Physics Division, Hsinchu, Taiwan \\ ${ }^{5}$ Department of Physics, University of Alberta, Edmonton, Alberta, Canada
}

(Received 27 December 2016; published 22 February 2017)

\begin{abstract}
We study, by first-principles computer simulations, the low-temperature phase diagram of bosonic dipolar gases in a bilayer geometry as a function of the two control parameters, i.e., the in-plane density and the interlayer distance. We observe four distinct phases, namely, paired and decoupled superfluids, as well as a crystal of dimers and one consisting of two aligned crystalline layers. A direct quantum phase transition from a dimer crystal to two independent superfluids is observed in a relatively wide range of parameters. No supersolid phase is predicted for this system.
\end{abstract}

DOI: 10.1103/PhysRevA.95.023622

\section{INTRODUCTION}

Quantum assemblies of particles featuring permanent electric or magnetic dipole moments are of interest for the intriguing, novel many-body physical effects that the anisotropic character of the interaction may underlie [1,2]. In the simplest physical setting, a gas of dipolar bosons is confined to two dimensions (2D), their dipoles all aligned perpendicularly to the plane by means of an external field; in this case, the interaction between two particles is purely repulsive, decaying as $1 / r^{3}$ at long distances, neither short nor quite long ranged. Experimentally, a realization of such a system is possible with molecules [3], ultracold Rydberg-excited atoms [4], and ultracold bosonic gases of dysprosium [5] confined to 2D by means of an external harmonic trap. The ground-state $(T=0)$ phase diagram of such a system has been studied by Monte Carlo simulations [6-10], yielding evidence of a first-order quantum phase transition between a superfluid and a crystal at high density.

Of great interest is also the case of a bilayer geometry, i.e., with dipolar particles (obeying either Fermi or Bose statistics) confined to two parallel planes. In this case, if dipoles are aligned as described above, the in-plane interaction is purely repulsive, while that between particles in different planes is attractive at short distances. The control parameters of this system, in the $T \rightarrow 0$ limit, are the in-plane density (or, equivalently, the mean interparticle distance $r_{s}$ ), assumed here to be the same for both planes, and the interlayer distance $d$.

The effect on the physics of the system of the interaction between particles in different layers depends on both $d$ and $r_{s}$ in a nontrivial way. In the two opposite limits $d \gg r_{s}$ and $d \ll r_{s}$, one expects the physics to be the same as that of a single layer, in the first case because the two layers decouple, in the second because the attraction between particles in different layers leads to the formation of increasingly tightly bound pairs (dimers), acting like dipolar bosons of twice the mass

\footnotetext{
*cinti@sun.ac.za

†cdwwang@phys.nthu.edu.tw

${ }^{\ddagger}$ m.boninsegni@ualberta.ca
}

and dipole moment of the original particles [11-14]. On the other hand, in the intermediate regime in which $d \approx r_{s}$, one may expect novel phases to occur as a result of the competition between the repulsive in-plane and the (mostly) attractive outof-plane interactions.

In many respects, one can regard such a bilayer system as an ideal playground to gain a general understanding of the physics of composite particles (CPs), ubiquitous in condensed matter (one need only think of Cooper pairs, polarons, excitons, composite fermions, or Feshbach molecules) as well as in nuclear physics (hadrons) [15]. For example, it is clearly relevant to the physics of excitons, which are bosonic CPs expected to undergo Bose-Einstein condensation (BEC) at low temperature. In spite of tremendous experimental effort in the last decades [16], unambiguous observation of excitonic BEC is still elusive [17]. It is also worthwhile mentioning that recent experimental advances in controlling ultracold dipolar atoms such as dysprosium [5] or erbium [18] are paving the way to novel experiments on bi- or multilayer geometries able to mimic the $\mathrm{CP}$ physics here discussed.

The ground-state phase diagram of dipolar bosons in a 2D bilayer geometry (in continuous space) has been studied by quantum Monte Carlo simulations $[19,20]$ at low in-plane density, where no crystallization occurs. In this paper, we carry out a comprehensive study of the low-temperature phase diagram of the system by means of quantum Monte Carlo simulations.

At low density, a $T=0$ quantum phase transition (previously observed by other authors [19]) occurs when the interlayer spacing $d$ is sufficiently small compared to the interparticle distance $r_{s}$ (roughly $d / r_{s} \lesssim 0.5$ ); specifically, two decoupled 2D superfluids (hereafter referred to as $2 \mathrm{SF}$ ) transition into a phase featuring short-range pairing correlations between nearest-neighboring particles in different layers. This phase, henceforth referred to as PSF (paired superfluid phase), has the character of a gas of tightly bound pairs (dimers) in the $d \rightarrow 0$ limit; in the vicinity of the transition, on the other hand, pairing is more loosely defined, and the distinction between PSF and 2SF in the $T \rightarrow 0$ limit rests on the different superfluid properties (see below). 
(a) Paired superfluid (PSF)

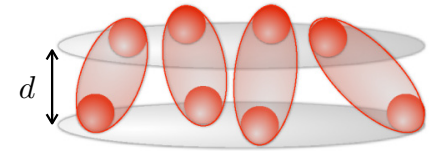

(c) Independent superfluids (2SF)
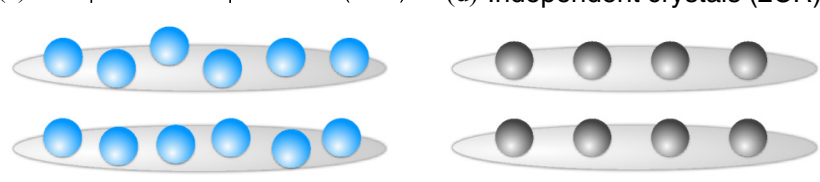

FIG. 1. Phases of a bilayer bosonic dipolar system. There are two superfluid phases, one consisting of a single superfluid of dimers (a), the other one comprising two independent 2D superfluids (b); (c) shows a crystal of dimers, while (d) two aligned crystal layers.

Increasing the density while holding $d$ constant has the effect of weakening the effective interlayer interaction, as a result of which the $2 \mathrm{SF}$ phase gains strength, extending to lower values of $d$ before transitioning into a dimer crystal (PC). Finally, in the high-density limit superfluidity disappears, and the PSF is replaced at large $d$ by two independent crystals (2CR), which are "locked" into an aligned arrangement as a result of potential energy minimization, a fact already noticed by other authors [21] in the classical limit of Eq. (1). All of these phases are schematically shown in Fig. 1 . At exactly $T=$ 0 , the two crystalline phases are structurally indistinguishable. However, their melting behavior at finite temperature is physically distinct, as will be illustrated below. No supersolid phase is observed, consistent with the observation, repeatedly made in recent times, that a softening of the repulsive pairwise interaction is a necessary ingredient for the appearance of such a phase [22-28].

The remainder of this paper is organized as follows: In Sec. II we discuss the model and the methodology, with particular emphasis on the calculation of the cogent quantities (mainly the superfluid density), and in Sec. III we illustrate our results. We outline our conclusions and discuss possible experimental observation of the phases described here in Sec. IV.

\section{MODEL AND METHODOLOGY}

We consider an ensemble of $2 N$ Bose particles of spin zero, mass $m$, and dipole moment $D$, confined to either one of two parallel planes at a distance $d$ from one another. Each plane contains $N$ particles, a number that is fixed, i.e., there is no physical mechanism whereby particles can "hop" from one plane to the other; all dipole moments are aligned in the direction perpendicular to the planes.

The Hamiltonian of the system in dimensionless units is the following:

$$
\begin{aligned}
\hat{H}= & -\frac{1}{2} \sum_{i, \alpha} \nabla_{i, \alpha}^{2}+\sum_{i \neq j, \alpha} \frac{1}{\left|\mathbf{r}_{i, \alpha}-\mathbf{r}_{j, \alpha}\right|^{3}} \\
& +\sum_{i, j} U_{d}\left(\mathbf{r}_{i 1}, \mathbf{r}_{j 2}\right)
\end{aligned}
$$

with

$$
U_{d}\left(\mathbf{r}, \mathbf{r}^{\prime}\right)=\frac{\left|\mathbf{r}-\mathbf{r}^{\prime}\right|^{2}-2 d^{2}}{\left(\left|\mathbf{r}-\mathbf{r}^{\prime}\right|^{2}+d^{2}\right)^{5 / 2}},
$$

where $\mathbf{r}_{i, \alpha}$ is the position of the $i$ th particle (dipole) of layer $\alpha=1,2$. All lengths are expressed in terms of the characteristic length of the dipolar interaction, namely, $a \equiv$ $m D^{2} / \hbar^{2}$, whereas $\epsilon \equiv\left(D^{2} / a^{3}\right)=\hbar^{2} /\left(m a^{2}\right)$ is the unit of energy and temperature (i.e., we set the Boltzmann constant $k_{B}=1$ ). The two control parameters of the Hamiltonian (1) in the $T \rightarrow 0$ limit are the layer distance $d$ and the mean interparticle distance $r_{s}=\left(n a^{2}\right)^{-1 / 2}$, where $n$ is the in-plane (2D) density [29].

The low-temperature phase diagram of the system described by Eq. (1) has been studied in this work by means of first-principles numerical simulations, based on the continuous-space worm algorithm [30,31]. Since this technique is by now fairly well established and extensively described in the literature, we shall not review it here. Details of the simulation are standard. In particular, we use a square cell with periodic boundary conditions in the two directions. The short imaginary time $(\tau)$ propagator utilized here is the usual one [32], accurate to order $\tau^{4}$; all of the results presented here are extrapolated to the $\tau \rightarrow 0$ limit. Numerical results shown here pertain to simulations with a number of particles $N$ on each layer between 36 and 144 .

Because we are mainly interested in the physics of the system in the $T \rightarrow 0$ limit, we generally report here results corresponding to temperatures $T$ sufficiently low to regard them as essentially ground-state estimates. A quantitative criterion to assess whether the temperature $T$ of the simulation is sufficiently low consists of monitoring the behavior of specific physical quantities as a function of $T$. In the superfluid phase, we consider "ground-state" estimates obtained at temperatures for which the computed superfluid fraction is within $\sim 5 \%$ of its extrapolated $T=0$ value (as explained below, this depends on the phase which one is considering); in the (nonsuperfluid) crystalline phase, the results that we furnish correspond to a temperature $T \lesssim 10^{-2}\langle K\rangle,\langle K\rangle$ being the kinetic energy per particle. However, we also discuss the behavior of the system as a function of temperature, notably, the superfluid transitions and the melting of the crystal phases.

As stated above, the number of particles $N$ in each plane is constant, i.e., there is no physical mechanism allowing for interplane hopping; thus the dipolar gases in the two planes are regarded as separate components, which requires the use of two separate "worms" [31], an especially important device in the study of paired superfluid phases.

The use of a finite-temperature technique to investigate what is essentially ground-state physics might appear counterintuitive, considering that methods exist in principle purposefully designed to study the ground state of a many-body system (e.g., diffusion Monte Carlo). In practice, however, finite-temperature techniques typically prove superior in the investigation of Bose systems, even to determine ground-state properties. This is mainly owing to the unbiasedness of finitetemperature methods, which, unlike their $T=0$ counterparts, require no a priori physical input (e.g., a trial wave function) and are not affected by additional bias coming from, e.g., the finite size of the population of random walkers, like diffusion 


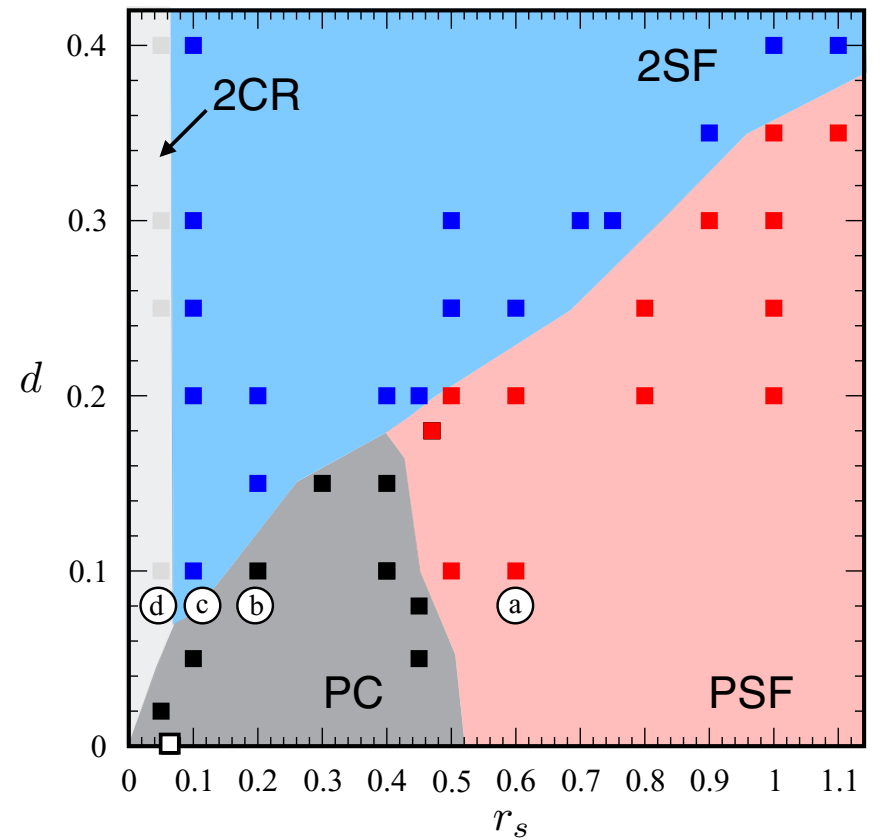

FIG. 2. Schematic ground-state phase diagram of bosonic dipolar gases on a bilayer geometry as a function of the interparticle distance $r_{s}$ and the interlayer separation $d$. Boxes refer to actual simulation results. 2SF stands for two decoupled 2D superfluids, PSF 2D pair superfluid, PC for a pair crystal, and 2CR for two separate 2D crystals. (a)-(d) Points for which pair-correlation functions are shown in Fig. 3. The open square along the $d=0$ line represents the single-layer crystal-superfluid phase transition as estimated in Ref. [9]

Monte Carlo [33,34]. Moreover, finite-temperature methods allow one to assess more easily and reliably quantities other than the energy, including off-diagonal correlations.

As mentioned above, we compute the superfluid fraction of the system as a function of temperature, using the well-known "winding number" estimator [35]. In this case, it is necessary to distinguish between two types of superfluid phases (of the three that are known to occur in two-component Bose mixtures [36]), one in which superflow takes place independently in the two planes (top left in Fig. 1), the other in which a superfluid of dimers occurs (top right in Fig. 1) [37]. The two phases can be distinguished simply through the value of the in-plane superfluid fraction $\rho_{S}(T)$, which saturates to $100 \%$ in the $T \rightarrow$ 0 limit if two decoupled superfluids exist (one in each plane), but to $50 \%$ in the presence of a superfluid of dimers, as a result of the twofold mass increase arising from the formation of the two-particle bound states.

\section{RESULTS}

Figure 2 offers an overview of the phase diagram, with its four distinct phases, which we now discuss. We restricted our study to the $r_{s} \leqslant 1$ region.

\section{A. Superfluid phases}

At low density $\left(r_{s} \gtrsim 0.5\right)$, the system displays a $2 \mathrm{D}$ superfluid character, but the nature of the phase changes as the layers are brought sufficiently close. Specifically, if the layers
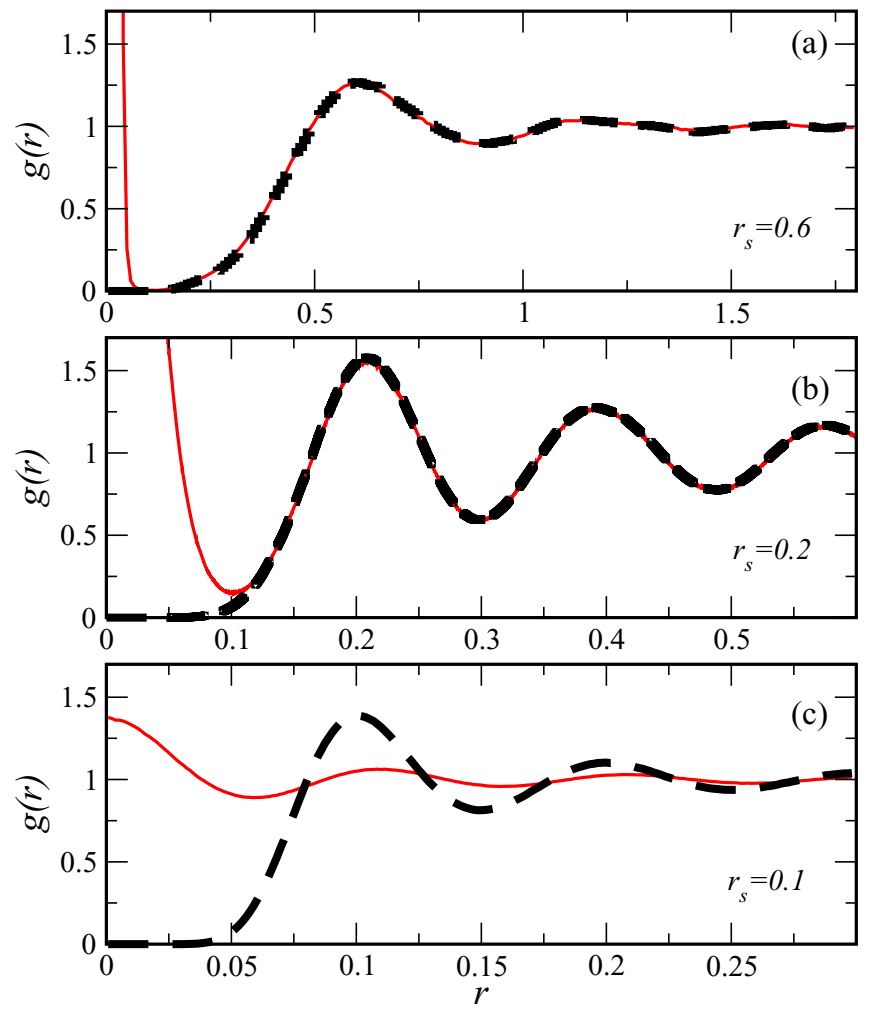

FIG. 3. Pair-correlation functions $g_{\alpha \alpha}(r)$ (black dashed line), $g_{\alpha \beta}(r), \alpha \neq \beta$ (solid line), with $\alpha, \beta=1,2$ plane indices. The interlayer distance $d=0.1$. Panel (a) refers to the pair superfluid phase (PSF) with $r_{s}=0.6$, (b) to the pair crystal (PC) with $r_{s}=0.2$, and (c) to two independent superfluids (2SF) with $r_{s}=0.1$. All of the curves shown here are representative of the $T=0$ limit.

are far apart $\left(d \gtrsim 0.4 r_{s}\right)$, the system features two decoupled 2D superfluid gases (2SF in Fig. 2). On the other hand, for close interlayer distances the short-range attractive well of the dipolar interaction between particles in different layers is deep enough that bound states of particles in different layers form, and what one observes is a 2D superfluid phase of dimers (PSF in Fig. 2).

The two different regimes can be identified through the value of the in-plane superfluid density $\rho_{S}(T)$, which, as explained above, saturates to $100 \%$ in the 2 SF phase but to $50 \%$ in the PSF one, as a result of the doubling of the mass of each particle, which affects its diffusion in imaginary time. Their different structure can be assessed through the calculation of the pair-correlation functions $g_{\alpha \beta}(r)$, where $\alpha, \beta=1,2$ are plane indices and where $r$ is a 2D distance.

Figure 3 shows the $g_{\alpha \alpha}(r)$ and $g_{\alpha \beta}(r), \alpha \neq \beta$, paircorrelation functions pertaining to the thermodynamic points indicated as (a), (b), and (c) in Fig. 2, i.e., $d=0.1$ in all cases. They are computed at sufficiently low temperature to be representative of the ground state of the system, i.e., the results do not change significantly, on the scale of the figures, if $T$ is further reduced.

The $g_{\alpha \alpha}$ and $g_{\alpha \beta}$ shown in panel (a) are essentially identical, except near the origin where $g_{\alpha \beta}$ (solid line) displays a strong peak, to signal the occurrence of a fluid phase of tightly bound pairs, with a short-range repulsion between pairs (as shown 
by both $g(r)$ going to zero at distance $\left.\sim r_{s} / 2\right)$. These are the pair-correlation functions characterizing the PSF phase. Panel (c) of Fig. 3 shows instead the 2SF phase; here, the in-plane pair-correlation function is that typical of a hard-core fluid, with a broad main peak at $r=r_{s}$ followed by rapidly decaying oscillations at greater distances. Meanwhile, the corresponding function for particles in different planes only features a modest enhancement near the origin, on account of the attraction between particles in different layers when they are on top of one another, but otherwise is nearly constant, to indicate that the superfluid dipolar gases in the two layers are decoupled. In other words, the main physical difference between 2SF and PSF is the existence in the latter of strong short-range correlations, which are always present in the PSF phase even when pairs are loosely bound. The character of the quantum phase transition between the PSF and the 2SF has been thoroughly discussed in Ref. [38], in which a study of a lattice version of Hamiltonian (1) was carried out.

The superfluid transition of both the 2SF and the PSF conforms to the 2D Berezinskii-Kosterlitz-Thouless (BKT) paradigm $[39,40]$. While this is expected in the 2SF case, as it has already been verified for the single-layer case [41], in the PSF regime the binding energy of a pair, of order $1 / d^{3}$ in our units, is a few times the characteristic BKT superfluid transition temperature $T_{B K T}$, which is of the order of $1 / r_{s}^{2}$. Thus the system transitions to a normal fluid of pairs at finite $T$, dissociation occurring at higher $T$.

It is worth noting that the physical behavior of the system in the superfluid part of the phase diagram is not independent of $r_{s}$. Specifically, while in the regime considered here (i.e., $r_{s}<$ 1) the physics of is that of a 2D quasi-BEC of (relatively) tightly bound pairs, in the $r_{s} \gg 1$ limit (not investigated here) the binding energy of the dimers decreases exponentially $[42,43]$ with the interlayer distance $d$; thus the spatial size of the dimer wave function can become comparable to the interparticle distance, and the physics of the system approaches that of a BCS superconductor.

In the vicinity of the 2SF/PSF quantum phase transition, the peak at the origin of the $g_{\alpha \beta}$ correlation function in the PSF phase tends to get weaker in the $T \rightarrow 0$ limit, due to both quantum exchanges and zero-point motion; however, within the range of density considered in this work, in no case do we observe thermal reentrance of the PSF phase (described, e.g., in Ref. [36]). However, the method utilized in this work does not allow us to exclude a fundamental change of the character of the phase at temperatures unattainable in practice, given the current computational resources. In any case, we note that this result seems consistent with the findings of Ref. [38]. Indeed, the main difference between our results and theirs is that no supersolid phase is observed in the continuum, reflecting an important, intrinsic difference between lattice and continuum Hamiltonians [26]. Indeed, the lattice version of (1) features a supersolid phase even on a single layer [44].

\section{B. Crystal phases}

For $r_{s} \lesssim 0.5$ and sufficiently low $d\left(d \lesssim 0.5 r_{s}\right)$, a crystalline phase of dimers arises (PC in Fig. 2). The paircorrelation functions for this phase are shown in Fig. 3(b). There is a feature in common with the PSF phase, namely,

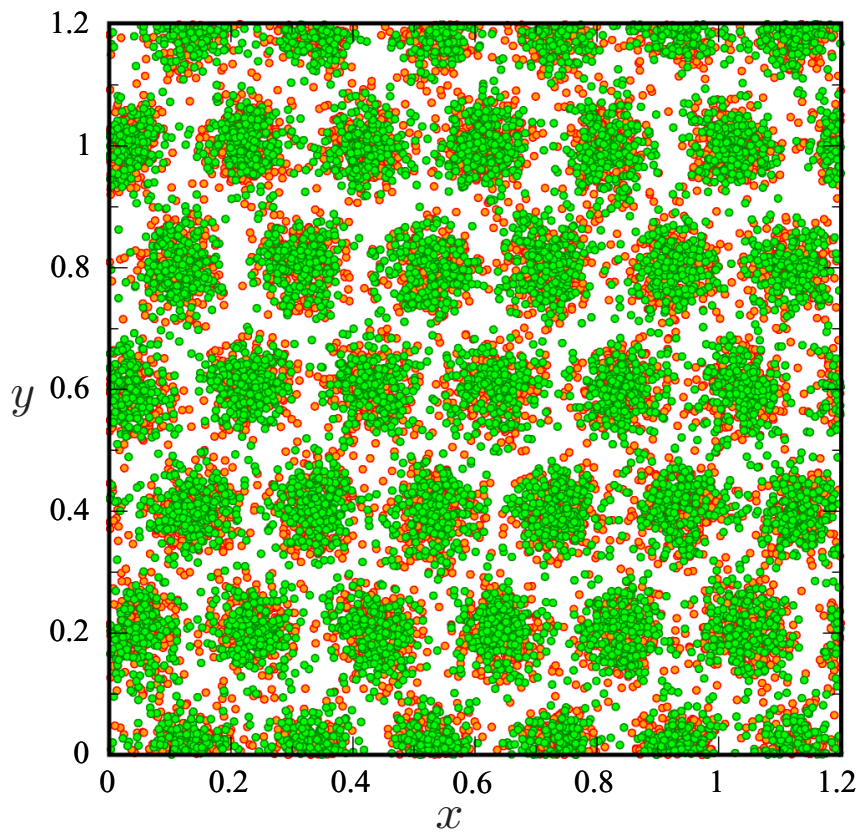

FIG. 4. Snapshot of many-particle configurations (world lines) for a bilayer system with mean interparticle distance $r_{s}=0.2$ and interlayer separation $d=0.1$, at temperature $T=0.1$ in the units adopted here (see text). Different colors refer to particles in different layers.

the strong peak at the origin (solid line) and the fact that the two $g(r)$ are on top of one another for distances greater than $\sim 0.5 r_{s}$. Unlike those of the PSF phase, however, the pair-correlation functions for this case display the persistent, marked oscillations that are typical of the crystalline phase. Just like for the PSF phase, the strong peak at the origin indicates the formation of tightly bound pairs. Crystallization can be rather easily detected by visual inspection of the many-particle configurations generated in the course of a simulation; a typical example is shown in Fig. 4, displaying an instantaneous snapshot of particle world lines. It is important to note that the crystalline arrangement shown in Fig. 4 occurs spontaneously, i.e., it is not initially imposed at the beginning of the simulation; the fact that particles in the two layers align nearly perfectly makes only one of the two layers clearly visible. The factor 8 difference between the freezing density of the single-layer system and that of the dimer one in the $d \rightarrow 0$ limit is a consequence of the doubled particle mass and fourfold increase of the strength of the dipolar coupling, as each dipole is doubled.

As $r_{s} \rightarrow 0$, two main physical effects occur, i.e., (a) the in-plane repulsive interaction increases, driving the system toward crystallization in each plane, and (b) the interlayer interaction is weakened. The weakening takes place as particles in one layer increasingly feel the effect of the nearest-neighboring particles in the other layer, as opposed to only (or, mainly) the one directly above, as is the case at low density. The overall result is that of a softening of the interlayer interaction, if the distance $d$ is kept constant.

The most important physical consequence is a strengthening of the 2SF phase, which progressively extends its domain 


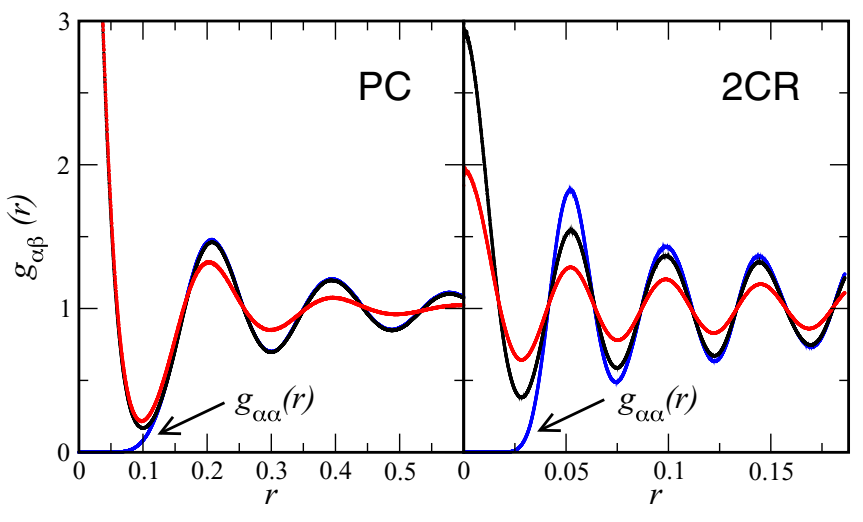

FIG. 5. Pair-correlation function $g_{\alpha \beta}(r), \alpha \neq \beta$ at different temperatures, for $d=0.1$. Left: $r_{s}=0.2$, and $T=1,100$ in the units utilized in this work. Right: $r_{s}=0.05, T=40,125$. Lower peaks correspond to higher $T$. Also shown is the corresponding $g_{\alpha \alpha}(r)$ at the lowest temperature for each case (arrows). In the results shown in the left panel, $g_{\alpha \alpha}$ cannot be distinguished from $g_{\alpha \beta}$ at the same temperature, for $r \gtrsim 0.1$.

of stability at low temperature to lower values of $d$, until $r_{s}$ reaches a value for which in-plane freezing into a triangular lattice begins to occur (as shown in Fig. 2). This value has been recently accurately estimated to be close to $r_{s}=0.064$ [9]. Our simulation confirms in-plane crystallization for this value of $r_{s}$, essentially independently of $d$, for $d \gtrsim 0.1$. The crystals in the two layers are "locked" into a configuration in which each particle in one layer sits above one in the other layer, as this minimizes the potential energy. We refer to this crystalline phase, which is physically distinct from the $\mathrm{PC}$ one, as consisting of two independent $2 \mathrm{D}$ crystals (2CR).

In the $T \rightarrow 0$ limit, the 2CR and PC phases are structurally indistinguishable. Neither the pair-correlation functions nor snapshots like that shown in Fig. 4 show any qualitative or quantitative differences. Rather, as observed also in Ref. [21], it is the behavior of the system at finite $T$ that allows one to draw a physically meaningful distinction between the two crystalline phases.

This is illustrated in Fig. 5 , which shows the temperature behavior of the pair-correlation function $g_{\alpha \beta}, \alpha \neq \beta$, for two different cases, corresponding to PC (left) and 2CR (right) ground states. The left panel shows $g_{\alpha \beta}$ for $d=0.1$ and $r_{s}=$ 0.2 , at the two temperatures $T=1,100$ in the units utilized here; the right panel shows results for $d=0.1, r_{s}=0.05$, and $T=40,125$. In the results shown in the left panel, the $g_{\alpha \alpha}$ and $g_{\alpha \beta}$ at the two temperatures are virtually indistinguishable, for $r \gtrsim r_{s} / 2=0.1$; at shorter distance, the $g_{\alpha \beta}$ develops a peak, as particles in different layers line up, whereas the $g_{\alpha \alpha}$ vanishes as a result of the in-plane, hard-core repulsion of the dipolar interaction. As the temperature is raised, both the $g_{\alpha \alpha}$ and $g_{\alpha \beta}$ lose structure, as the crystal order characterizing the ground state progressively disappears, but they remain indistinguishable above $r_{s} \sim r_{s} / 2$. This is consistent with the melting of a system of tightly bound dimers.

Let us now examine the very different behavior shown in the right panel. Here, too, as expected, in the $T \rightarrow 0$ limit, $g_{\alpha \beta}$ and $g_{\alpha \alpha}$ become identical for $r \gtrsim 0.025=r_{s} / 2$ and display the same features as the curves in the left panel for $r \lesssim r_{s} / 2$; however, as the temperature is raised, the $g_{\alpha \beta}$ quickly loses structure, while the $g_{\alpha \alpha}$ changes very little in the temperature range shown. Visual inspection also confirms that crystalline order persists in both planes. However, at finite temperature the simulated, finite-size crystals in the two layers can shift with respect to one another as a result of the weak interlayer potential energy of attraction, which causes the $g_{\alpha \beta}$ to be almost "flat" (i.e., nearly constant at a value unity) at a temperature $T=250$. All of this shows that the physics of the system is essentially that of two independent layers. The melting of the in-plane crystal takes place at considerably higher temperature than those shown in the right panel of Fig. 4.

Returning to the ground-state phase diagram of Fig. 2, an interesting feature arising from the competing effects of in-plane repulsion and out-of-plane attraction is the presence of a region, e.g., $0.05 \leqslant r_{s} \leqslant 0.2, d=0.1$, inside which, on increasing the density (i.e., $r_{s} \rightarrow 0$ ), first the dimer crystal quantum melts into two decoupled superfluids, which then successively crystallize again into the $2 \mathrm{CR}$ phase. At $T=0$, the PC phase can quantum melt into a PSF or $2 \mathrm{SF}$, as the layers are moved away from one another, or transitions into $2 \mathrm{CR}$. It is worth mentioning that the stability of the 2SF phase, near the 2CR and PC phase boundaries (e.g., $r_{s}=0.1, d=0.1$, see Fig. 2), is crucially underlain by quantum-mechanical exchanges; indeed, simulations treating particles as distinguishable yield a stable PC phase in a considerably more extended region of the phase diagram, as already remarked in previous works, for dipolar Bose systems [45].

We conclude this section by discussing the melting of the PC and 2CR phases. Our simulations show that the PC phase always melts into a dimer fluid, either normal or superfluid depending on the density. In particular, in the $d \rightarrow 0$ limit, when the dimers are strongly bound, the physics of the system reproduces that of a single layer, which is of course also approached in the $d>>r_{s}$ limit, the only difference between the two regimes being a rescaling of the unit of length by a factor 8, as explained above. We obtained in this work numerical evidence of melting of the single-layer system into a superfluid, close to the $T=0$ melting density (i.e., $r_{s} \approx 0.5$ ). On the other hand, the $2 \mathrm{CR}$ phase is always found to melt into two independent normal fluid phases on the two layers.

\section{CONCLUSIONS}

In this work, we have employed exact numerical methods to investigate the low-temperature phase diagram of dipolar bosons in a bilayer geometry, all dipoles aligned perpendicularly to the planes. In the two opposite limits in which the in-plane mean interparticle distance $r_{s}$ is either much less or much greater than the interlayer separation $d$, the physics of the system is that of a single-layer system [6,9]. On the other hand, as a result of the competition between the in-plane (repulsive) and the out-of-plane (attractive) interactions, the intermediate regime $\left(d \approx r_{s}\right)$ gives rise to considerably more complex and interesting physics. In particular, at low density $\left(r_{s} \gtrsim 0.5\right)$ a quantum phase transition occurs as $d \lesssim r_{s} / 2$ from a phase consisting of two independent superfluid $2 \mathrm{D}$ gases to a superfluid phase of bound pairs of particle in different layers. No reentrant behavior of either phase was observed at finite $T$. 
At higher density, we observe two distinct solid phases, physically related to the superfluid ones, namely, one of tightly bound dimers, which arises when the interlayer separation is less than the mean interparticle distance, and one comprising two independent 2D crystalline layers. An interesting feature of the phase diagram is the direct transition of the system from a crystalline phase of dimers into one of independent 2D superfluids, which is observed in a rather wide range of parameters.

In terms of possible experimental realization, one can estimate characteristic physical values for the parameters of Eq. (1), e.g., by considering a realistic polar molecule, say $\mathrm{SrO}$, for which $a \approx 122.2 \mu \mathrm{m}$ for a fully polarized state with
$D=8.9$ D. Considering that both the interlayer and mean interparticle distances are of the order of a fraction of a $\mu \mathrm{m}$ in typical experiments, one can easily imagine tuning $r_{s}$ and $d$ in a rather wide range.

\section{ACKNOWLEDGMENTS}

We acknowledge fruitful discussion with F. Mezzacapo, A. Kuklov, B. Capogrosso-Sansone, J.-S. You, and Y.-Y. Tian. This work was supported in part by the Natural Science and Engineering Research Council of Canada. Computing support of Westgrid is gratefully acknowledged. D.D.W. is supported by research grant of NCTS and MoST in Taiwan.
[1] M. A. Baranov, Phys. Rep. 464, 71 (2008).

[2] T. Lahaye, C. Menotti, L. Santos, M. Lewenstein, and T. Pfau, Rep. Prog. Phys. 72, 126401 (2009).

[3] M. H. G. de Miranda, A. Chotia, B. Neyenhuis, D. Wang, G. Quéméner, S. Ospelkaus, J. L. Bohn, J. Ye, and D. S. Jin, Nat. Phys. 7, 502 (2011).

[4] See, for instance, T. G. Gallagher, Rydberg Atoms (Cambridge University Press, Cambridge, 1994).

[5] H. Kadau, M. Schmitt, M. Wenzel, C. Wink, T. Maier, I. FerrierBarbut, and T. Pfau, Nature (London) 530, 194 (2016).

[6] H.-P. Büchler, E. Demler, M. Lukin, A. Micheli, N. Prokofev, G. Pupillo, and P. Zoller, Phys. Rev. Lett. 98, 060404 (2007).

[7] G. E. Astrakharchik, J. Boronat, I. L. Kurbakov, and Yu. E. Lozovik, Phys. Rev. Lett. 98, 060405 (2007).

[8] C. Mora, O. Parcollet, and X. Waintal, Phys. Rev. B 76, 064511 (2007).

[9] S. Moroni and M. Boninsegni, Phys. Rev. Lett. 113, 240407 (2014).

[10] P. Jain, F. Cinti, and M. Boninsegni, Phys. Rev. B 84, 014534 (2011).

[11] D.-W. Wang, M. D. Lukin, and E. Demler, Phys. Rev. Lett. 97, 180413 (2006).

[12] D.-W. Wang, Phys. Rev. Lett. 98, 060403 (2007).

[13] W. Lechner and P. Zoller, Phys. Rev. Lett. 111, 185306 (2013).

[14] A. K. Fedorov, S. I. Matveenko, V. I. Yudson, and G. V. Shlyapnikov, Sci. Rep. 6, 27448 (2016).

[15] See, for example, B. R. Martin, Nuclear and Particle Physics (John Wiley \& Sons, New York, 2006).

[16] L. V. Butov, C. W. Lai, A. L. Ivanov, A. C. Gossard, and D. S. Chemla, Nature (London) 417, 47 (2002); A. A. High, J. R. Leonard, M. Remeika, L. V. Butov, M. Hanson, and A. C. Gossard, Nano Lett. 12, 2605 (2012).

[17] Recent progress for quantum Hall excitons may have some new development, but full many-body theory in the high-density regime seems still unclear. See J. P. Eisenstein and A. H. MacDonald, Nature (London) 432, 691 (2004); P. Bhattacharya, B. Xiao, A. Das, S. Bhowmick, and J. Heo, Phys. Rev. Lett. 110, 206403 (2013); C. Schneider et al., Nature (London) 497, 348 (2013).

[18] L. Chomaz, S. Baier, D. Petter, M. J. Mark, F. Wächtler, L. Santos, and F. Ferlaino, Phys. Rev. X 6, 041039 (2016).

[19] A. Macia, G. E. Astrakharchik, F. Mazzanti, S. Giorgini, and J. Boronat, Phys. Rev. A 90, 043623 (2014).

[20] A. Filinov, Phys. Rev. A 94, 013603 (2016).
[21] X. Lu, C.-Q. Wu, A. Micheli, and G. Pupillo, Phys. Rev. B 78, 024108 (2008).

[22] F. Cinti, P. Jain, M. Boninsegni, A. Micheli, P. Zoller, and G. Pupillo, Phys. Rev. Lett. 105, 135301 (2010).

[23] S. Saccani, S. Moroni, and M. Boninsegni, Phys. Rev. B 83, 092506 (2011).

[24] S. Saccani, S. Moroni, and M. Boninsegni, Phys. Rev. Lett. 108, 175301 (2012).

[25] M. Boninsegni, J. Low Temp. Phys. 168, 137 (2012).

[26] M. Boninsegni and N. Prokof'ev, Rev. Mod. Phys. 84, 759 (2012).

[27] F. Cinti, T. Macrì, W. Lechner, G. Pupillo, and T. Pohl, Nat. Commun. 5, 3235 (2014).

[28] M. Boninsegni, J. Low Temp. Phys. 184, 1071 (2016).

[29] We are obviously implicitly assuming that the layer width is much smaller than both $r_{s}$ and $d$, i.e., the layers can be regarded as very nearly $2 \mathrm{D}$.

[30] M. Boninsegni, Nikolay Prokofev, and B. Svistunov, Phys. Rev. Lett. 96, 070601 (2006).

[31] M. Boninsegni, N. V. Prokofev, and B. V. Svistunov, Phys. Rev. E 74, 036701 (2006).

[32] See, for instance, M. Boninsegni, J. Low Temp. Phys. 141, 27 (2005).

[33] M. Boninsegni and S. Moroni, Phys. Rev. E 86, 056712 (2012).

[34] M. Boninsegni, Phys. Rev. Lett. 111, 235303 (2013).

[35] E. L. Pollock and D. M. Ceperley, Phys. Rev. B 36, 8343 (1987).

[36] A. Kuklov, N. Prokof'ev, and B. Svistunov, Phys. Rev. Lett. 92, 030403 (2004).

[37] No phases featuring "super-counterflow" are observed here.

[38] A. Safavi-Naini, S. G. Söyler, G. Pupillo, H. R. Sadeghpour, and B. Capogrosso-Sansone, New J. Phys. 15, 013036 (2013).

[39] V. L. Berezinskii, Sov. Phys. JETP 32, 493 (1971).

[40] J. Kosterlitz and D. Thouless, J. Phys. C: Solid State Phys. 6, 1181 (1973).

[41] A. Filinov, N. V. Prokofev, and M. Bonitz, Phys. Rev. Lett. 105, 070401 (2010).

[42] B. Simon, Ann. Phys. (NY) 97, 279 (1976).

[43] A. Pikovski, M. Klawunn, G. V. Shlyapnikov, and L. Santos, Phys. Rev. Lett. 105, 215302 (2010).

[44] L. Pollet, J. D. Picon, H.-P. Büchler, and M. Troyer, Phys. Rev. Lett. 104, 125302 (2010).

[45] M. Boninsegni, L. Pollet, N. Prokof'ev, and B. Svistunov, Phys. Rev. Lett. 109, 025302 (2012). 